\title{
CALIDAD PERCIBIDA EN CENTROS DE ATENCIÓN TEMPRANA DE ANDALUCÍA. ESTUDIO CUALITATIVO
}

\section{The perceived quality of Early Intervention Centers in Andalusia. A qualitative study}

\author{
Rita Pilar Romero Galisteo \\ Universidad de Málaga. Facultad de Ciencias de la Salud. Departamento de Fisioterapia \\ Manuel Lillo CRESPO \\ Universidad de Alicante. Facultad de Enfermería. Departamento de Enfermería. Campus San \\ Vicente del Raspeig. Alicante \\ manuel.lillo@grupohla.com \\ Sofía García SAN JuAN \\ Universidad de Alicante. Facultad de Enfermería. Departamento de Enfermería
}

Recepción: 10 de octubre de 2018

Aceptación definitiva: 7 de noviembre de 2018

Resumen: No abundan las publicaciones sobre Centros de Atención Temprana y todavía menos las referentes a calidad percibida por sus usuarios y familias. La falta de consenso en la organización de estos servicios a nivel regional, nacional e internacional, así como el desconocimiento social de los mismos y su función, justificarían la necesidad de ahondar en este campo. El objetivo de este estudio fue explorar la calidad de servicio percibida por parte de las familias atendidas en Centros de Atención Temprana de Andalucía desde una perspectiva cualitativa, a través de entrevistas con familias mediante cuestionario escrito. Del análisis surgieron 2 categorías: lo que funciona (fortalezas) y lo que no funciona (debilidades) y 6 subcategorías relacionadas con: aspectos temporales, espaciales, estructurales, organizacionales, de recursos humanos y resultado de las terapias. Como conclusión se aprecia que la mayor debilidad identificada radica en elementos tangibles del servicio. Por contra, los aspectos relacionales son mejor valorados por las familias. Conocer la calidad en los servicios de Atención Temprana permite compatibilizar con éxito los objetivos tanto de usuarios como de los centros, introduciendo 
así una importante mejora en la valoración del impacto en la salud de las familias y niños atendidos en este tipo de centros.

Palabras clave: Atención Temprana; familia; calidad percibida; estudio cualitativo.

AвSTRAct: Publications are not abundant about Early Childhood Intervention Centers and even less with reference to the perceived quality of their users and families. The lack of consensus in the organization of these services at the regional, national and international levels, as well as the lack of its social recognition and its target population, can contribute partially to this lack of knowledge. The objective of this study was to explore the perceived quality of service by the families attended in the Early Intervention Centers in Andalusia from a qualitative perspective, using interviews with parents through written questionnaires. Two categories merged during the analysis: what works well (strengths) and not so well (weaknesses) that derived in 6 subcategories regarding the organization, allocation, timing, structure, human resources and therapies' outcomes. As main conclusions, the weaknesses identified lied in tangible elements of the service while, professional-family relational aspects were better valued by families. To know the quality of Early Intervention Centers could allow us improving the population attention by matching the objectives of both users and institutions, thus introducing positive changes in the assessment of the impact on the families' and children's health.

KEY wORDs: Early Intervention; family; perceived quality; qualitative study.

\section{Introducción}

utores como Guralnick definen la Atención Temprana (AT) como un sistema de atención diseñado para apoyar patrones familiares de interacción que mejor estimulen el desarrollo evolutivo del niño, cuando existe en el mismo algún déficit o riesgo de padecerlo (Guralnick, 2001). Distintas investigaciones se han referido a la importancia del desarrollo temprano en el desarrollo posterior del niño(European Agency for Development in Special Needs Education, 2005). En España, se entiende por AT el conjunto de intervenciones, dirigidas a la población infantil de 0-6 años, a la familia y al entorno, que tienen por objeto dar respuesta lo más pronto posible a las necesidades transitorias o permanentes que presentan los niños con trastornos en su desarrollo o que tienen el riesgo de padecerlos (GAT, 2005). Atendiendo y cubriendo las necesidades y demandas de las familias y del entorno en el que vive el niño se cumple uno de los objetivos de la AT.

La infancia es un período de vulnerabilidad en el cual los factores genéticos y familiares interactúan con las exposiciones ambientales y otros determinantes que llevan a una compleja combinación de efectos y resultados en salud (Rajmil, Díaz y Peiró, 2011). Entre los diagnósticos habituales que pueden atenderse en AT destacan los trastornos en el desarrollo motriz, cognitivo, del lenguaje, sensorial, de la conducta, evolutivo, prematuridad y niños en riesgo psicosocial, entre otros (Robles-Bello y Sánchez-Teruel, 2013). El hecho de que se trate a población infantil y con ese tipo de 
diagnóstico puede estar relacionado con la falta de visibilidad social y consecuentemente de evaluación de la calidad de los centros.

La organización de la AT no es homogénea en Europa, sin embargo, todos los países ofrecen servicios dirigidos a la población infantil en riesgo biológico, psicológico o social y a sus familias. A diferencia de los EE. UU., donde la AT comenzó dirigiéndose a niños en situación de desventaja socioeconómica, en Europa, la AT nació vinculada a la discapacidad, situación que ha ido cambiando con los años (Ponte, 2005). Ejemplos a destacar son Grecia, Suiza o Portugal, donde se atiende a niños entre 0 y 6 o 7 años que sufren algún problema físico-orgánico-funcional, así como a los que están en riesgo de sufrir retraso en el desarrollo; Austria, Alemania o Reino Unido, donde la AT se presenta como apoyo sociosanitario específico; Malta y Noruega ofrecen AT hasta los 6 años. Como se aprecia, no existe un modelo único, aunque sí puede observarse la transición de un modelo biomédico hacia uno social. A pesar de las diferencias, la tendencia generalizada en Europa va encaminada hacia la descentralización de los servicios, es decir, ubicar los Centros de АT (САT) tan cerca como sea posible del niño y su familia. Asimismo, en todos los países se cuenta con un equipo multidisciplinar del ámbito sanitario, educativo y/o social que incluye desde médicos, enfermeros o educadores infantiles hasta psicólogos, trabajadores sociales, fisioterapeutas y que varía según el país. Los modelos de financiación combinan fondos estatales con iniciativas locales público-privadas que ofertan AT a todos los niños con necesidad y sus familias con independencia de su situación socioeconómica (European Agency for Development in Special Needs Education, 2005).

En España no existe una Ley estatal de AT, siendo las distintas autonomías las que legislan en esta materia. Esta situación refleja la heterogeneidad de la AT en nuestro país. Así, Castilla León y Navarra prestan AT en los CAT hasta los 3 años. Por otro lado, salvo en Ceuta y Melilla, en el resto del territorio nacional no es necesaria la condición de discapacidad para recibir AT como prestación de servicio. Del mismo modo, existen comunidades con legislación específica (Aragón, Castilla-La Mancha, Cataluña, Extremadura, Galicia, Cantabria, Castilla y León, La Rioja y Valencia) y otras en las que se está legislando de manera precisa en los últimos años, como p. e.: Andalucía, Madrid, Murcia y Canarias (Quirós Pérez, 2009; Robles-Bello y SánchezTeruel, 2013). Respecto a la financiación, la situación no difiere del resto de Europa. Las iniciativas políticas defienden claramente los servicios de AT. La financiación pública de la oferta de servicios de AT combina fondos procedentes del Gobierno central, fondos regionales, locales, así como de organizaciones sin ánimo de lucro (p. e.: asociaciones de síndrome de Down, sordera, autismo, etc.). Ocasionalmente puede dotarse a las familias de fondos en forma de becas para el uso de servicios privados, aunque esto es algo puntual (European Agency for Development in Special Needs Education, 2005; GAT, 2011).

A pesar de existir diferencias en el territorio nacional, debidas a las políticas de descentralización y a la historia propia de cada comunidad autónoma, existe consenso en cuanto a la definición, objetivos y ámbitos de actuación. La intervención, por ejemplo, considera la globalidad del niño y es planificada por un equipo de profesionales de orientación interdisciplinar, siendo habituales psicólogos, logopedas y 
fisioterapeutas, aunque pueden implicarse también trabajadores sociales, pediatras, psicopedagogos, etc. (GAT, 2005; GAT, 2011; Robles-Bello y Sánchez-Teruel, 2013). En este sentido, en España, la AT está viviendo un proceso de transformación en el que se empiezan a conocer experiencias que muestran la transición desde un modelo de intervención más clínico a uno más social en el que familia y entorno cobran especial relevancia (Escorcia Mora, García-Sánchez, Orcajada Sánchez y Sánchez López, 2016).

Resulta necesario destacar la consideración actual de la familia como eje fundamental en la atención infantil (Guralnick, 2011; Hadders-Algra, Tjitse y Hamer, 2016; Mas, Basqués, Balcells-Ballcells, Dalmau, Giné, Grácia et al., 2016).

La incorporación de la opinión del usuario al funcionamiento de los sistemas sanitarios no es algo nuevo (Pilgrim, Cardona, Pinder y Sonenstein, 2014). El desarrollo de mecanismos que evalúen la oferta y aseguren que la calidad de los estándares es respetada mejoraría la eficacia de los servicios de AT y garantizarían la misma calidad de la oferta nacional de los servicios a pesar de las diferentes idiosincrasias (Agencia Europea para el desarrollo de la educación del alumnado con necesidades educativas especiales, 2010). Donabedian (1966) consideró la satisfacción del paciente como una medida del resultado de la asistencia sanitaria. Por otro lado, la calidad del servicio percibida consiste en dar o superar lo que el cliente espera y se enfatiza en que no depende únicamente de los aspectos técnicos, sino, especialmente, de los corporativos (Gulc, 2017; Mira, Rodríguez-Marin, Tirado y Sitges, 2000). Existe consenso en considerar satisfacción y calidad del servicio como constructos diferentes aunque relacionados (Alén González y Fraiz Brea, 2006).

Conviene recordar que, tradicionalmente, la percepción de los consumidores sobre la calidad del servicio ha servido como referencia para mejorar los programas $(\mathrm{Ca}-$ labrese y Scoglio, 2012; Dunst y Trivette, 2005; García, Salanova, Grau y Cifre, 2013; Romero-Galisteo, Morales-Sánchez y Hernández-Mendo, 2015). Esta perspectiva se centra en los juicios de los consumidores sobre la calidad técnica de los servicios, así como en percepciones más subjetivas de las experiencias con los profesionales que proporcionan el servicio (Bruder y Dunst, 2015; Gabbott y Hogg, 1994; Seth, Deshmukh y Vrat, 2005; Zeithaml, 1998). Por otro lado, la calidad del servicio se ha evaluado habitualmente en términos de satisfacción y lealtad (Duffy, 2005; Sierra y McQuitty, 2005). Sin embargo, estas serían el resultado o la consecuencia de la calidad del servicio, según algunos expertos en consumo (Oliver, 1993).

Tras las conclusiones obtenidas en un estudio previo realizado con la misma muestra, en el que se validó una herramienta para medir de forma fiable la calidad percibida, se llegó a la conclusión de que la perspectiva de las familias podría resultar útil para mejorar la calidad de los propios CAT (Romero-Galisteo et al., 2015), por lo que parece necesario indagar más en este constructo. En esta línea, autores como García-Grau, McWilliam, Martínez Rico y Morales Murillo (2018) apuntan a que la calidad de vida familiar podría ser un buen indicador de la calidad del servicio. Estos nuevos resultados de la investigación posiblemente avalen cambios importantes en los próximos años en los modelos de prestación de servicios a las personas con discapacidad intelectual o del desarrollo (Giné, Montero Centeno, Verdugo Alonso, Rueda Quillet y Vert Tapia, 2015).

(C) Ediciones Universidad de Salamanca / CC BY-NC-ND

Siglo Cero, vol. 50 (2), n. ${ }^{\circ}$ 270, 2019, abril-junio, pp. 7-18 
La calidad es un valor añadido a la prestación de cualquier servicio y, aunque es un término que todos los consumidores conocen, no suele concretarse adecuadamente (Martínez Fuentes y Martínez Hernández, 2013). El objetivo de este estudio fue explorar la calidad percibida por parte de las familias atendidas en los CAT desde una perspectiva cualitativa.

\section{Método}

Se trata de un diseño de corte cualitativo, por ser el más indicado para el objetivo de estudio (Paley, 1997), que tiene que ver con la experiencia vivida por las familias en los contextos donde se lleva a cabo la Atención Temprana. Sin embargo, el desconocimiento de estas unidades hace que las propias familias no dispongan de una experiencia previa o visión para establecer comparación de forma natural.

\subsection{Participantes}

La muestra total se compuso de 887 padres y madres de usuarios de los diferentes CAT ubicados en Málaga y su provincia. Se realizó un muestreo no aleatorio por selección intencionada donde los criterios de inclusión fueron que las familias decidieran participar de manera anónima y voluntaria y que llevaran más de un mes asistiendo a sesiones de AT, para que conocieran el funcionamiento del centro. Como único criterio de exclusión se estableció que los participantes no comprendieran o pudieran expresarse en castellano.

El $70.7 \%$ de la muestra $(n=628)$ corresponde a madres y el $21.7 \%(n=193)$ a padres de los niños atendidos en los distintos centros participantes. Respondieron a la pregunta abierta del cuestionario 195 usuarios, lo que supone un $21.98 \%$ del total de la muestra.

\subsection{Instrumento de recogida de datos}

Este estudio forma parte de una investigación más amplia en la que se validaron y analizaron las propiedades psicométricas de un cuestionario que evalúa la calidad del servicio percibida en los Centros de Atención Temprana (Romero-Galisteo et al., 2015). La recogida de datos se realizó a través de un documento escrito que se empleó en la investigación anteriormente mencionada y, para la parte cualitativa, se incluyó una pregunta abierta sobre la calidad del centro al final de dicho cuestionario. En este estudio se ha trabajado únicamente con los datos aportados en esta última parte del cuestionario, ya que la finalidad del mismo se centra en explorar el constructo calidad del servicio desde un enfoque cualitativo. 


\subsection{Contexto de estudio}

Puesto que nuestro objeto de estudio se centró en Andalucía, es necesario explicar brevemente la situación en dicha comunidad debido a la variabilidad que, como se ha mencionado, existe. En Andalucía la AT depende de la Consejería de Salud desde el año 2005. De esta manera, ante la sospecha de trastorno de desarrollo o riesgo de padecerlo, desde cualquier ámbito (educativo, social y sanitario), será el pediatra de Atención Primaria quien inicie el trámite de derivación a las Unidades de Atención Infantil Temprana. Estas se componen de un psicólogo y otro pediatra, quienes tras determinar la necesidad de intervención lo derivarán a un Centro de Atención Infantil Temprana.

\subsection{Procedimiento}

La administración del cuestionario contó con el consentimiento y aceptación de colaboración de los diferentes centros participantes y cuenta con la aprobación del Comité de Ética de la Universidad de Málaga (n. ${ }^{\circ}$ registro: IF246-15). Previamente, hubo una primera toma de contacto tanto con un asesor técnico como con la consultora de AT del Servicio de Planificación y Evaluación Asistencial de la Delegación Provincial de Salud de la Junta de Andalucía en Málaga, que facilitaron los datos administrativos y manifestaron la necesidad, el interés y la pertinencia de este tipo de estudios, y a su vez actuaron como informantes privilegiados y pieza clave en la revisión del análisis cualitativo de la información recopilada. La cumplimentación del cuestionario se realizó de manera individual por el familiar que acompañaba en ese momento al niño a la terapia correspondiente. En la mayoría de los centros, se llevó a cabo en la sala de espera de los mismos, es decir, sin la presencia de cualquiera de los profesionales que integraban equipos de los distintos CAT participantes.

\subsection{Análisis de datos}

La validez en investigación cualitativa está presente tanto en el proceso sistemático de obtención como en el análisis de los datos (Paley, 1997). Para tratar los datos, se utilizó el análisis de contenido (Andréu Abela, 2000). Las respuestas fueron analizadas por dos investigadores con experiencia en análisis cualitativo, que no participaron en la recolección de los datos previa, y uno que sí participó en ella con experiencia en este tipo de metodología de estudio. Estuvieron en contacto y se reunieron para consensuar la información. El análisis de datos también fue refutado por los informantes privilegiados conocedores de los contextos. Posteriormente, se agruparon en categorías, extrayendo finalmente los temas que se presentan a continuación a modo de resultados. 


\section{Resultados}

En el análisis aparecieron 2 temas principales: 1) lo que funciona o aspectos positivos (fortalezas) y 2) lo que no funciona o aspectos negativos (debilidades) que a su vez se explican a través de 6 subcategorías utilizando el proceso descrito anteriormente. De los aspectos negativos del análisis también se extrajeron propuestas de mejora. En la Tabla 1 se muestran los temas y sus componentes constituyentes o subtemas.

\begin{tabular}{|l|l|}
\hline \multicolumn{2}{|c|}{ TABLA 1. Temas y subtemas surgidos en el análisis } \\
\hline \multicolumn{1}{|c|}{ Temas } & \multicolumn{1}{|c|}{ SubTemas } \\
\hline Lo que no funciona + propuestas de mejora & $\begin{array}{l}\text { Aspectos temporales } \\
\text { Aspectos espaciales/estructurales } \\
\text { Aspectos organizacionales } \\
\text { Aspectos del recurso bumano }\end{array}$ \\
\hline Lo que funciona & $\begin{array}{l}\text { Recurso bumano } \\
\text { Resultados de las intervenciones }\end{array}$ \\
\hline
\end{tabular}

\subsection{Tema 1: Lo que no funciona + propuestas de mejora}

\subsubsection{Aspectos temporales}

Los usuarios hacen constantes referencias al escaso tiempo de las sesiones, tanto en su duración como en la distancia entre ellas: P25 "Deberían durar más, y no ser sólo una a la semana”. Además también hacen referencia a la tardanza entre la sesión de valoración y las de continuación, apuntando además una falta de coordinación entre lo que ocurre entre sesiones por parte de diferentes profesionales: P10 “Tardaron más de dos meses en que mi hijo empezara la terapia”. Por otro lado, también se pone de manifiesto que el fin de la terapia esté en función de unos protocolos y no de las necesidades de los niños: P30 “Deberían seguir hasta que hiciera falta y no sólo hasta los cinco años".

\subsubsection{Aspectos espaciales/estructurales}

Los usuarios del centro hicieron constantes alusiones a las deficiencias de las infraestructuras del centro, como la dificultad de acceso, la falta de climatización, el mobiliario poco adecuado o incluso cierta dejadez en el cuidado de la sala de espera: P03 "Debería haber mejor aparcamiento"; P10 "La sala de espera no está bien para los niños”. Además creían necesario ser conocedores de los avances de sus hijos y poder realizar sugerencias y/o encuestas de satisfacción: P15 "Estaría bien que hubiera un buzón de sugerencias”; P07 “Me gustaría que pudiera recibir información on-line”. 


\subsubsection{Aspectos organizacionales}

Los participantes en este estudio mostraron su preocupación por la falta de coordinación entre los centros de salud, los colegios y el centro. Llevando esto, a una falta de coordinación e información con las propias familias: P24 "Estaría bien que el pediatra pudiera saber lo que están haciendo los niños"; P11 "Que el colegio se pusiera en contacto con el centro". También creían que existía mucha masificación de niños y pocas ayudas por parte de la Junta de Andalucía, que además impedía la ampliación de una cartera de servicios: P05 "Muchos niños por hora”; P13 "Se necesitan más ayudas, las sesiones son muy caras”.

\subsubsection{Aspectos del recurso humano}

En cuanto al personal, los usuarios del centro hablan de poca formación de la mayoría de los profesionales que trabajan allí, además de falta de cobertura cuando se encuentran de baja laboral los titulares y demasiados cambios de profesionales por contratos muy cortos: P27 "Deberían hacer más cursos de formación"; P18 "En un año hemos tenido tres fisioterapeutas distintos, no deberían cambiar tanto”.

\subsection{Tema 2: Lo que funciona}

\subsubsection{Recurso humano}

A pesar de la poca profesionalización que han detectado, sí se muestran muy contentos con el trato que los profesionales profesan a sus hijos, siendo este humano, cercano y cariñoso. Y valoran el esfuerzo realizado por los centros por la cartera de personal especializado: P20 "Los terapeutas son muy cariñosos"; P19 "Hay fisioterapeutas, logopedas, terapeutas... aunque a veces son muy pocos y no llegan”.

\subsubsection{Resultados de las terapias}

En general, y a pesar de todas las cosas que piensan que se pueden mejorar, la mayoría de los usuarios están satisfechos con los resultados de las terapias, ya que califican los resultados con el aumento de la autoestima y la confianza de sus hijos: P17 "Desde que viene al centro está mucho más centrado"; P08 "En general le van muy bien, ha mejorado mucho desde que empezó”.

\section{Discusión}

En este estudio se detallan las percepciones de los padres sobre la calidad del servicio que reciben sus hijos en los CAT. El análisis cualitativo realizado pone de manifiesto 
las percepciones de las familias participantes contribuyendo de esta manera a aumentar el conocimiento sobre el tema y, por ende, servirá para subsanar las debilidades observadas en los CAT analizados. En él se hace referencia a los procesos de mejora, incrementando la eficacia, la competitividad y la satisfacción de los clientes, aportando garantías mínimas para lograr la competitividad necesaria para su subsistencia y mantenimiento.

Autores como Piskur et al. (2012) afirman que se conoce poco sobre las necesidades de los niños con discapacidad y de sus familias y que ahondar en ello es esencial para futuras investigaciones.

La mayoría de las sugerencias hacen referencia a la infraestructura física o instalaciones del CAT apreciándose, por tanto, las menciones a los elementos tangibles. La calidad relacional, en cambio, no ha sido tan ampliamente aludida, coincidiendo así con las aportaciones de autores como Ödman, Krevers y Öberg (2007), que afirman que los padres perciben mayor calidad de servicio en función de la mejor relación de los profesionales con sus hijos y no tanto en relación con la mejora que puedan aportar las terapias recibidas. Si bien en el estudio sobre calidad de servicio de Gracia, Vidal-Sellés y Martínez-Tur (2017) los participantes son las propias personas con discapacidad intelectual, los resultados de nuestro trabajo apuntan en el mismo sentido. Es decir, la interacción con los profesionales, tanto en su faceta profesional o técnica (calidad funcional) como en la afectiva (calidad relacional), suele ser valorada positivamente, lo que favorecería tanto la satisfacción con el centro como el bienestar general de las familias atendidas.

Por otro lado, la mayoría de las percepciones de los pacientes son medidas con instrumentos de corte cuantitativo (Male, Noble, Atkinson y Marson, 2017), sin embargo, Porthé, Vargas, Ronda, Malmusi, Bosch y Vázquez (2018) añaden un tinte cualitativo a la evaluación de la calidad de los servicios sanitarios. De esta manera, analizando el discurso de los participantes, emergen los problemas descritos. En los CAT no ocurre de forma diferente si se incorpora el punto de vista de los actores principales, en este caso, las familias.

La mayoría de los estudios que han solicitado la participación de las familias para evaluar la calidad de los servicios lo han hecho desde una perspectiva cuantitativa (Aytch, Castro, Selz-Campbell y Laurie, 2004; Dyke, Buttigieg, Blackmore y Ghose, 2006; Kontos y Diamond, 2002; Epley, Summers y Turnbull, 2011; Romero-Galisteo, Gálvez-Ruiz, Belda-Oriola y Milla-Romero, 2015), siendo esta una de las escasas investigaciones cualitativas que analiza la calidad en AT y concretamente en España.

No en todos los países existen directrices que definan la calidad para los servicios de AT. En algunos países europeos existen medidas políticas especiales para controlar que los estándares de calidad sean respetados (Agencia Europea para el desarrollo de la educación del alumnado con necesidades educativas especiales, 2010). En España todavía se necesita dotar a los CAT de mayor peso específico y garantizar la calidad del servicio sin diferencias geográficas.

Generalizar las conclusiones de estos resultados es tal vez una limitación de este trabajo. Si bien extrapolar los datos de Andalucía al resto de España puede resultar arriesgado, este estudio supone una primera aportación al estudio de este constructo en el resto de CAT del territorio nacional e internacional. 
Por otra parte, investigar sobre la calidad de los servicios prestados en los CAT permite compatibilizar con éxito las expectativas de usuarios y los objetivos de los propios centros, en pro de las tendencias actuales relacionadas con el "paciente activo" y el "empoderamiento del usuario". De esta manera, tanto profesionales como gerentes tendrán datos fiables sobre sus fortalezas y debilidades sobre los que sustentar un plan de mejora continua.

\section{Referencias bibliográficas}

Agencia Europea para el Desarrollo de la Educación del Alumnado con Necesidades Educativas Especiales (2010). Atención Temprana. Progresos y Desarrollo 20052010. Odense, Dinamarca: Agencia Europea para el Desarrollo de la Educación del Alumnado con Necesidades Educativas Especiales.

Alén GonzÁlez, M. E. y Fraiz Brea, J. A. (2006). Relación entre la calidad de servicio y la satisfacción del consumidor. Su evaluación en el ámbito del turismo termal. Investigaciones Europeas de Dirección y Economía de la Empresa, 12 (1), 251-272.

ANDRÉU AbElA, J. (2000). Las técnicas de análisis de contenido: una revisión actualizada. Fundación Centro Estudios Andaluces. Universidad de Granada, 10 (2), 1-34. Recuperado de: http://public.centrodeestudiosandaluces.es/pdfs/S200103.pdf. Consulta: 2/10/2018.

Aytch, L., Castro, D. y Selz-Campbell y Laurie, M. S. (2004). Early Intervention Services Assessment Scale (EISAS): conceptualization and development of a program quality selfassessment instrument. Infants E Young Children, 17 (3), 236-246. doi: 10.1097/00001163200407000-00005.

Bruder, M. B. y Dunst, C. J. (2015). Parental Judgments of Early Childhood Intervention personnel practices: applying a consumer science perspective. Topics in Early Childhood Special Education, 34 (4), 200-210. doi: 10.1177/027112145414522527.

Calabrese, A. y Scoglio, F. (2012). Reframing the past: A new approach in service quality assessment. Total Quality Management and Business Excellence, 23, 1329-1343. doi: 10.1080/14783363.2012.733259.

Donabedian, A. (1966). Evaluating the quality of medical care. Millbank Memorial Fund Quarterly, 44 (2), 166-206.

Duffy, D. L. (2005). The evolution of customer loyalty strategy. Journal of Consumer Marketing, 22, 284-286.

Dunst, C. J. y TRIVETTE, C. M. (2005). Measuring and evaluating family support program quality. Asheville, NC: Winterberry Press.

Dyke, P., Buttigieg, P., Blackmore, A. M. y Ghose, A. (2006). Use of the Measure of Process of care for families and service providers to evaluate family-centered services in a pediatric disability setting. Child Care, Health and Development, 32 (2), 167-176.

Epley, P. H., Summers, J. y Turnbull, A. P. (2011). Familiy outcomes of Early Intervention: families' perceptions of need, services and outcomes. Journal of Early Intervention, 33 (3), 201-219. doi: 10.1177/1053815111425929.

Escorcia Mora, C. T., García-Sánchez, F. A., Orcajada Sánchez, N. y Sánchez López, M. C. (2016). Perspectiva de las prácticas de atención temprana centradas en la familia desde la logopedia. Revista de Logopedia, Foniatría y Audiología, 36 (4), 170-177. https://doi. org/10.1016/j.rlfa.2016.07.002. 
European Agency for Development in Special Needs Education (2005). Early Childhood Intervention. Analysis of Situations in Europe. Key Aspects and Recommendations. Summary Report. Recuperado de https://www.european-agency.org/sites/default/files/ early-childhood-intervention-analysis-of-situations-in-europe-key-aspects-and-recommendations_eci_en.pdf. Consulta: 2/10/2018.

Gaвbотt, M. y Hogg, G. (1994). Consumer behaviour and services: A review. Journal of Marketing Management, 10, 311-324. doi: 10.1080/0267257X.1994.9964277.

García, E., Salanova, M., Grau, R. y Cifre, E. (2013). How to enhance service quality through organizational facilitators, collective work engagement and relational service competence. European Journal of Work and Organizational Psychology, 22, 42-55. doi: 10.1080/135943X.2011.628793.

García-Grau, P., McWilliams, R. A., Martínez Rico, G. y Morales Murillo, C. P. (2018). Child, family and early intervention characteristics related to family quality of life in Spain. Journal of Early Intervention. doi: 10.1177/1053815118803772.

gat (Federación Estatal de Asociaciones de Profesionales de Atención Temprana) (2005). Libro Blanco de la Atención Temprana. Madrid: Ministerio de Trabajo y Asuntos Sociales. Real Patronato sobre Discapacidad.

gat (Federación Estatal de Asociaciones de Profesionales de Atención Temprana) (2011). La realidad actual de la Atención Temprana en España. Madrid: Real Patronato sobre Discapacidad.

Giné Giné, C., Montero Centeno, D., Verdugo Alonso, M. Á., Rueda Quitllet, P. y Vert TAPIA, S. (2015). Claves de futuro en la atención y apoyo a las personas con discapacidad intelectual y del desarrollo. ¿Qué nos dice la ciencia? [Keys to future in the care and support for people with intellectual and developmental disabilities. What tell us Science?]. Siglo Cero, 46 (1), 253, 81-106. http://dx.doi.org/10.14201/scero201546181106.

Gracia, E., Vidal-Sellés, N. y Martínez-Tur, V. (2017). La calidad de servicio desde el punto de vista de las personas con discapacidad intelectual: relaciones con su satisfacción y bienestar. [Service quality from the point of view of people with intellectual disability: relationships with its satisfaction and wellbeing]. Siglo Cero, 48 (3), 263, 41-53. http://dx.doi. org/10.14201/scero20174834153.

Gulc, A. (2017). Models and methods of measuring de quality of logistic service. Procedia Engineering, 182, 255-264. doi: 10.1016/j.proeng.2017.03.187.

GuralnicK, M. J. (2001). A developmental systems' model for Early Intervention. Infants and Young Children, 14 (2), 1-18.

Guralnick, M. J. (2011). Why Early Intervention works. A systems perspective. Infants and Young Chilren, 24 (1), 6-28.

Hadders-Algra, M., Tjitse Hielkema, A. G. B. y Hamer, E. G. (2016). Effect of early intervention in infants at very high risk of cerebral palsy: a systematic review. Developmental Medicine and Child Neurology, 59 (3), 246-258.

Kontos, S. y DiAmond, K. (2002). Measuring the quality of Early Intervention services form infants and toddlers: problems and prospects. International Journal of Disability, Development and Education, 49 (4), 337-351. doi: 10.1080/1034912022000028321.

Male, L., Noble, A., Atkinson, J. y Marson, T. (2017). Measuring patient experience: a systematic review to evaluate psychometric properties of patient reported experience measures (PREMS) for emergency care service provision. International Journal for Quality in Health Care, 29 (3), 314-326. doi: 10.1093/intqhc/mzx027.

Martínez Fuentes, M. T. y Martínez Hernández, L. (2013). Promoción del desarrollo infantil y atención temprana: calidad de los servicios. Azarbe: Revista Internacional de Trabajo Social y Bienestar, 2, 49-67.

() Ediciones Universidad de Salamanca / CC BY-NC-ND

Siglo Cero, vol. 50 (2), n. ${ }^{\circ}$ 270, 2019, abril-junio, pp. 7-18 
Mas, J. M., Basqués, N., Balcells-Balcells, A., Dalmau, M., Giné, C., Grácia, M. et al. (2016). Family quality of life for families in Early Intervention in Spain. Journal of Early Intervention, 38 (1), 59-74.

Mira, J. J., Rodríguez-Marín, J., Tirado, S. y Sitges, E. (2000). Semejanzas y diferencias entre satisfacción y calidad percibida. Revista Calidad Asistencial, 15, 36-42.

Ödman, P., Krevers, B. y ÖBerg, B. (2007). Parent's perceptions of the quality of two intensive training programmes for children with cerebral palsy. Developmental Medicine and Child Neurology, 49, 93-100.

Oliver, R. L. (1993). A conceptual model of service quality and service satisfaction: compatible goals, different concepts. En T. A. Swartz, D. E. Bowen y S. W. Brown (Eds.), Advances in services marketing and management: Reseearch and practice (pp. 65-85). Greenwich, CT: JAI Press.

Paley, J. (1997). Husserl, phenomenology and nursing. Journal of Advanced Nursing, 26 (1), 187-193. https://doi: 10.1046/j.1365-2648.1997.1997026187.x.

Pilgrim, N. A., Cardona, K. M., Pinder, E. y Sonenstein, F. L. (2014). Clients' perceptions of service quality and satisfaction at their initial Title $\mathrm{X}$ family planning visit. Health Communication, 29 (5), 505-515. doi: 10.1080/10410236.2013.777328.

Ponte, J. (2005). Proyectos europeos de Atención Temprana. En M. G. Milla y F. Mulas (Eds.), Atención Temprana. Desarrollo infantil, diagnóstico, trastornos e intervención (pp. 865-883). Valencia: Promolibro.

Porthé, V., Vargas, I., Ronda, E., Malmusi, D., Bosch, L. y Vázquez, M. L. (2018). Has the quality of health care for the immigrant population changed during the economic crisis in Catalonia (Spain)? Opinions of health professionals and inmigrant users. Gaceta Sanitaria, 32 (5), 425-432. doi.org: 10.1016/j.gaceta.2017.03.010.

Priskur, B., Beurskens, A. J. H. M., Jongmans, M. J., Ketelaar, M., Norton, M., Frings, C. A., Hemmingsson, H. y Smeets, R. J. E. M. (2012). Parent's actions, challenges and needs while enabling participation of children with a physical disability: a scoping review. BMC Pediatrics, 12, 177. doi: 10.1186/1471-2431-12-177.

Quirós Pérez, V. (2009). Nuevo modelo para la atención temprana en Andalucía: directrices y desafíos. Revista Iberoamericana de Educación, 48 (4), 1-8.

Rajmil, L., Díaz, E. y Peiró, R. (2011). Desigualdades sociales en la salud infantil. Informe SESPAS 2010. Gaceta Sanitaria, 24 (Supl.), 42-48.

Robles-Bello, A. y SÁnchez-Teruel, D. (2013). Atención Infantil Temprana en España. Papeles del Psicólogo, 34 (2), 132-143.

Romero-Galisteo, R. P., Gálvez-Ruiz, P., Belda-Oriola, J. C. y Milla-Romero, M. G. E. (2015). Percepción de la calidad en Centros de Atención Temprana: resultados de un análisis comparativo. Escritos de Psicología, 8 (2), 71-76. doi: 10.5231/psy.writ.20151206.

Romero-Galisteo, R. P., Morales-Sánchez, V. y Hernández-Mendo, A. (2015). Desarrollo de una herramienta para la evaluación de la calidad percibida en los centros de atención infantil temprana. Anales de Psicología, 31 (1), 127-36. doi: 10.6018/analesps.31.1.158191.

Seth, N., Deshmukh, S. G. y VRat, P. (2005). Service quality models: a review. International Journal of Quality and Reliability Management, 22, 913-949. doi: 10.1108/026567105106225211.

SiERRA, J. J. y McQuiTTY, S. (2005). Service providers and customers: social exchange theory and service loyalty. Journal of Services Marketing, 19, 392-400.

Zeithaml, V. A. (1998). Consumer perceptions of price, quality, and value: a means-end model and synthesis of evidence. Journal of Marketing, 52 (3), 2-22. doi: 10.2307/1251446. 